\title{
PMSM System Controlled by SVM-DTC
}

\author{
Weiwei Zhang ${ }^{1, a}$, Fei Xiao ${ }^{1}$ and Mingzhong Qiao ${ }^{2}$ \\ ${ }^{1}$ National Key Laboratory for Vessel Integrated Power System Technology, Navel University of Engineering, China \\ ${ }^{2}$ Department of Electrical Engineering, Navel University of Engineering, China
}

\begin{abstract}
Direct Torque Control (DTC), proposed after Vector Control (VC), has been widely used in speed regulation system due to its good dynamic performance. In order to solving the shortage of poor torque stationary properties of traditional DTC, this paper introduces direct torque control using space vector modulations (SVM-DTC) in Permanent Magnet Synchronous Motor (PMSM) system, which reduces torque ripple by using SVM and maintains fast dynamic response. Simulation model of SVM-DTC under MATLAB condition has been set up and compared with system controlled by traditional DTC. The results testify that the PMSM system based on SVM-DTC has high rate dynamic response, high stationary precision, and good robustness when load has a disturbance.
\end{abstract}

\section{Introduction}

Permanent magnet synchronous motor has been widely applied in various AC drive systems for its merits of high efficiency, high power density and high torque/inertia ratio. The research of permanent magnet synchronous motor drive system with high performance produces important economy and society value. While PMSM controlled by traditional DTC has some shortages because of using bangbang control. Only eight space vector voltage can be chosen lead to the ripple of stator flux and torque in this control. Thus, application of PMSM in high-precision occasion is limited [1].

In order to solve the problem of traditional DTC, many researches has been carried out recently. A number of methods such as space vector modulation, duty cycle control, vectors subdivision have been proposed and obtained some achievements [2]. The main idea of SVM is calculating exact vector voltage needed in every sampling period, so that SVM-DTC has many benefits in reducing ripple of torque except its hysteresis effect. Nevertheless, solutions in paper [3] and [4] have strong dependence on parameters of PMSM and are hard to control. In order to avoid ripple radically, paper [5] utilizes rotating coordinates to obtain definite vector voltage. However, the method depends on complex structure and hard to maintain the fast response of traditional DTC. The SVM-DTC presented in this paper aims to maintain the stationary performance and increase the speed of dynamic response substantially as well as the robustness.

\section{Analysis of Torque Ripple in Traditional DTC}

\footnotetext{
${ }^{a}$ Weiwei Zhang : zww_zhang@163.com 
Ignoring the core saturation, eddy current loss, hysteresis loss and rotor damper winding, the function of PMSM in $\alpha$ - $\beta$ coordinate system can be shown as (1) to (4).

$$
\begin{aligned}
& \psi_{\alpha}=\int\left(u_{\alpha}-r_{s} i_{\alpha}\right) d t \\
& \psi_{\beta}=\int\left(u_{\beta}-r_{s} i_{\beta}\right) d t \\
& \psi_{s}=\sqrt{\psi_{\alpha}^{2}+\psi^{2}} \beta \\
& T_{e}=\frac{3 n_{p}}{2 L_{s}}\left|\psi_{s}\right| \psi_{f} \sin \delta
\end{aligned}
$$

In the above formulas, $u_{\alpha}, i_{\alpha}, \psi_{\alpha}$ represent the stator voltage, stator current, and stator flux in $\alpha$ axis; $u_{\beta}, i_{\beta}, \psi_{\beta}$ represent the stator voltage, stator current, stator flux in $\beta$ axis; $r_{S}$ represents the stator resistance; $\psi_{s}$ represents the stator flux; $\psi_{f}$ represents the rotor flux; $T_{e}$ represents the electromagnetic torque; $L_{S}$ represents the stator inductance; $n_{p}$ represents the number of pole pairs; $\delta$ represents the angle of stator flux and rotor flux.

According to the relation of space vector with flux in traditional DTC (Figure 1), we conclude that only one vector can be chosen in one control cycle time, so that the ripple of torque can't be eliminated completely.

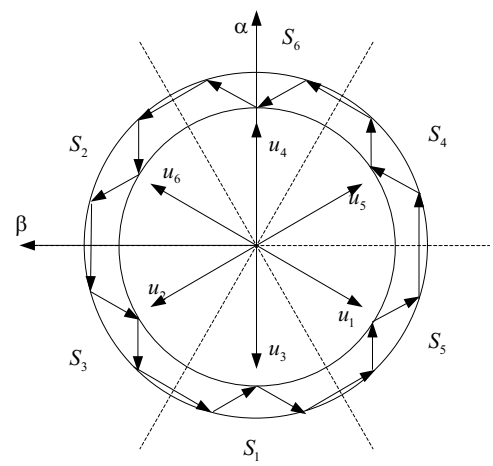

Figure 1. Relation of space vector with flux

\section{DTC Using Space Vector Modulations (SVM-DTC)}

\subsection{Shortage of Existing SVM-DTC}

Space vector generating of SVM-DTC proposed in paper [5] is showed in Figure 2. D-axis voltage component in d-q coordinate system can be obtained from PI output of the deviation compared actual flux with given flux; q-axis voltage component in d-q coordinate system can be obtained from PI output of the deviation compared actual torque with given torque. Compared with traditional DTC, SVM-DTC proposed in paper [5] overcoming the larger ripple of torque, while control structure becomes complicated due to introduction of rotating coordinate transformation, and hard to maintain 
the fast response. What's more, motor parameters and load change affecting PI performance lead to poor robustness.

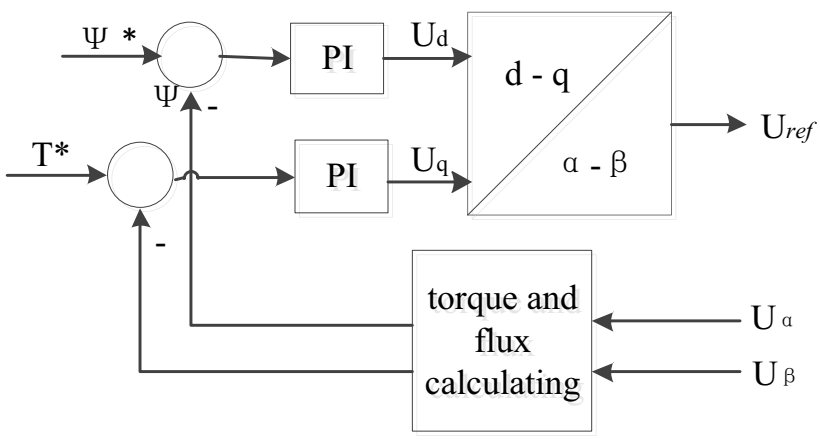

Figure 2. Generating of vector voltage in rotating coordinate

\subsection{SVM-DTC Proposed in This Essay}

In this paper, the effect of vector voltage on stator and rotor flux in one sampling period is shown in Figure 3. Relation between flux and voltage can be obtained only through mathematical analysis without complex rotating coordinate transformation [6].

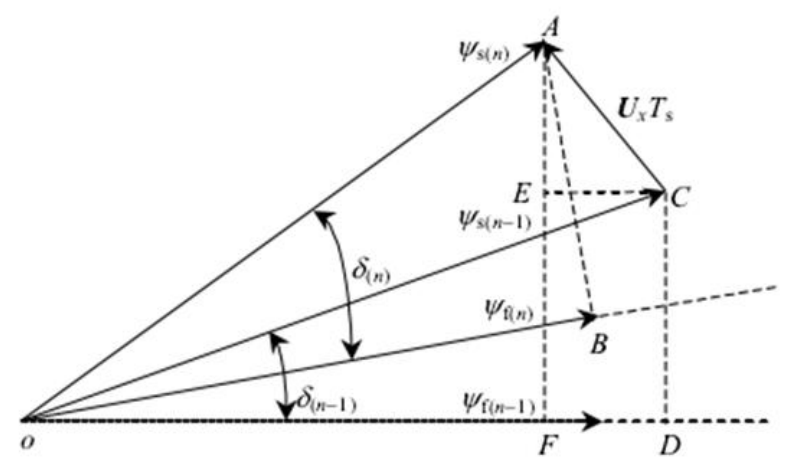

Figure 3. Flux before and after sampling period

By analyzing change of stator and rotor flux, accurate vector voltage can be calculated from functions expressed by the formula (5) to (9).

$$
\begin{gathered}
u_{\alpha r e f}=\frac{\psi_{s}^{*} \cos (\theta+\Delta \theta)-\psi_{s} \cos \theta}{T_{S}}+r_{s} i_{\alpha} \\
u_{\beta r e f}=\frac{\psi_{s}^{*} \sin (\theta+\Delta \theta)-\psi_{s} \sin \theta}{T_{S}}+r_{s} i_{\beta} \\
\Delta \theta=\Delta \theta_{r}+\delta(n)-\delta(n-1) \\
\Delta \theta_{r}=w_{r} \cdot T_{s} \\
\delta=\sin ^{-1}\left(\frac{2 T_{e} L_{s}}{3 n_{p} \psi_{s} \psi_{f}}\right)
\end{gathered}
$$


In the above formulas, $u_{\alpha r e f}, u_{\beta r e f}$ represent the accurate $\alpha$-axis voltage component and $\beta$ axis voltage component in $\alpha-\beta$ coordinate system; $\psi{ }_{S}^{*}$ represents the given stator flux; $\theta$ represents the angle between stator flux and $\alpha$-axis; $\Delta \theta$ represents the changed $\theta$ after one sampling period; $\delta(n), \delta(n-1)$ represents the angle between stator and rotor flux before and after sampling period; $w_{r}$ represents the rotor speed; $T_{S}$ represents the sampling cycle time; the remaining parameters definition is consistent with the previous text.

\subsection{Simulation Model}

On the basis of the previous analysis, simulation of PMSM system controlled by SVM-DTC is set up and represented in Figure 4.

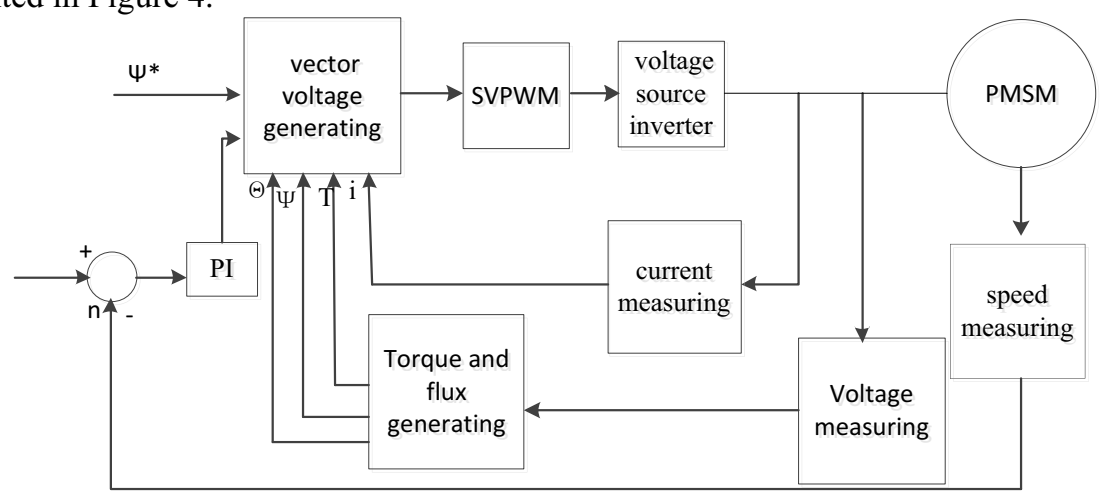

Figure 4. SVM-DTC simulation model

\section{Measurements and Simulation}

In order to verify the effectiveness of the control system proposed in this essay, simulation of traditional DTC and SVM-DTC in this paper have been done on the same PMSM model. Research of flux waveform and speed wave motion in steady state, torque ripple and load sudden change in dynamic is presented in this essay [7].

Figure 5(a) and 5(b) shows flux waveform controlled by traditional DTC and SVM-DTC in this essay when given stator flux is $0.8 w b$ in steady-state PMSM. The ripple in Figure 5(b) is obviously smaller than Figure 5(a).

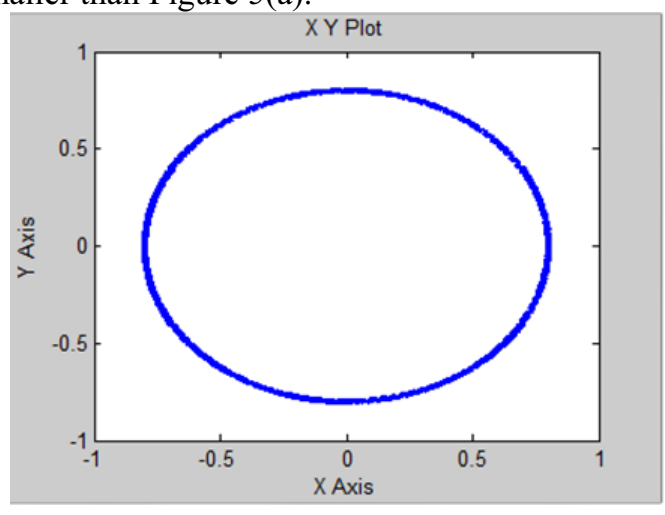

(a)Traditional DTC flux

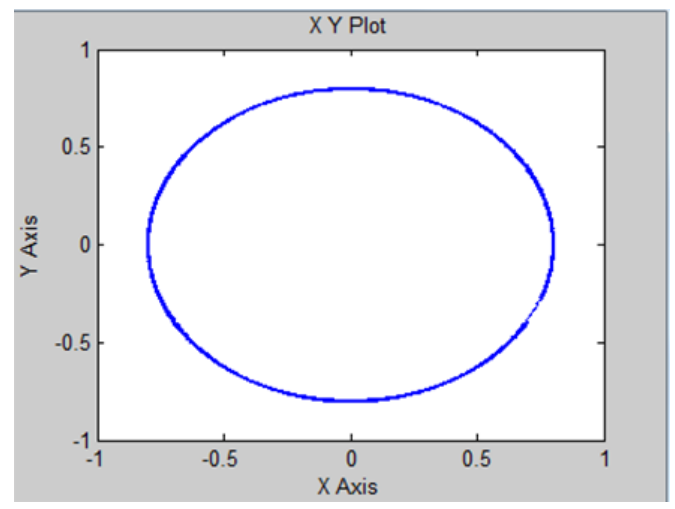

(b)SVM-DTC flux

Figure 5. Flux simulation results 
Figure 6(a) and 6(b) shows speed waveform controlled by traditional DTC and SVM-DTC in this essay when given speed is $1000 \mathrm{n} / \mathrm{rpm}$ in steady-state PMSM. The ripple in Figure 6(a) is about $\pm 4 n / r p m$, while it in Figure 6(b) is about $\pm 2 n / r p m$. Speed is more stable in SVM-DTC than traditional DTC.

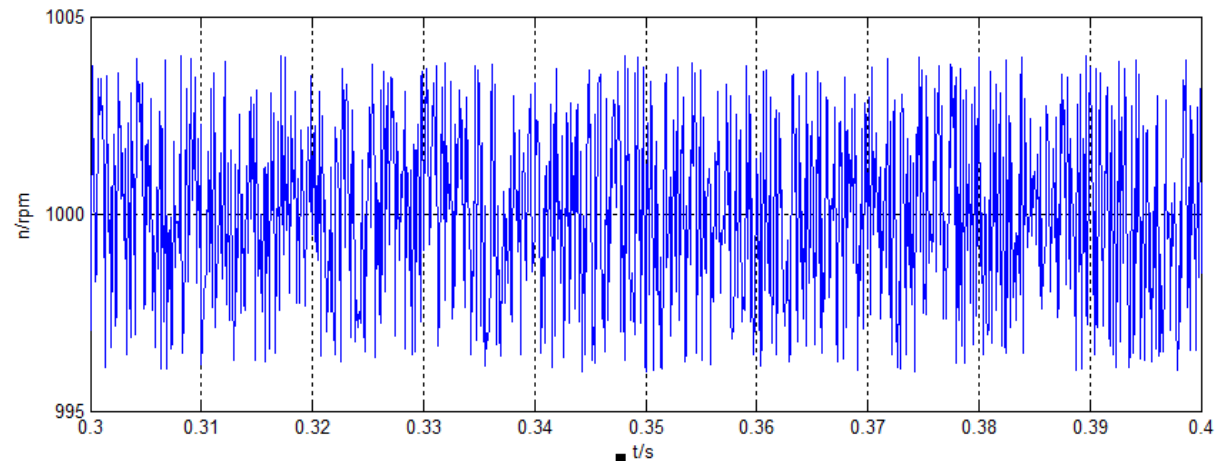

(a)Traditional DTC speed

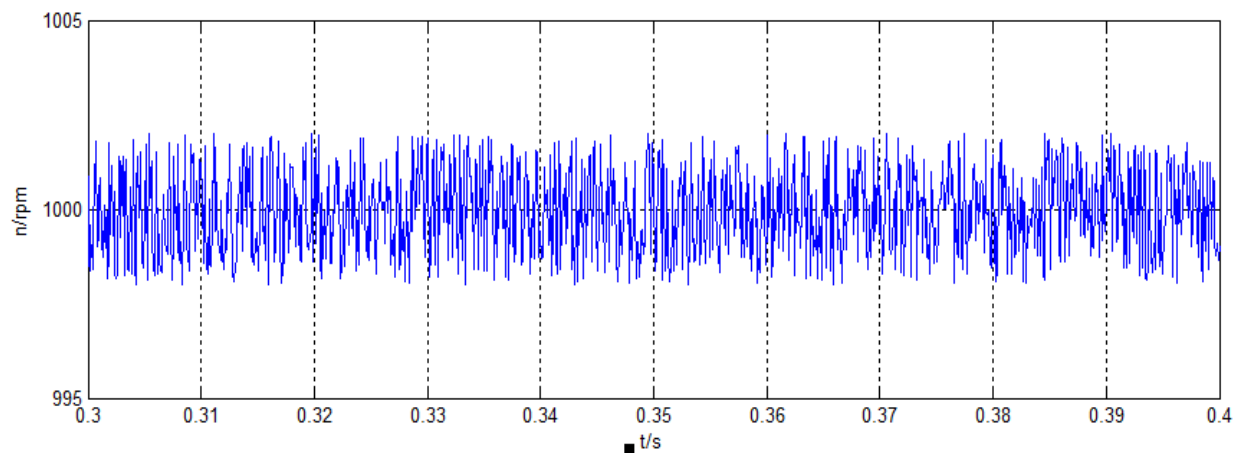

(b)SVM-DTC speed

Figure 6. Speed simulation results

For verifying the ripple of torque and dynamic performance, loads are changed in the same PMSM model controlled by different DTC. Load suddenly increases to 15 N.m from initial 5 N.m at 0.33 seconds and then dips back to 5 N.m at 0.37 seconds. The blue waveform represents torque of traditional DTC and the red waveform represents torque of SVM-DTC as displayed in Figure 7.

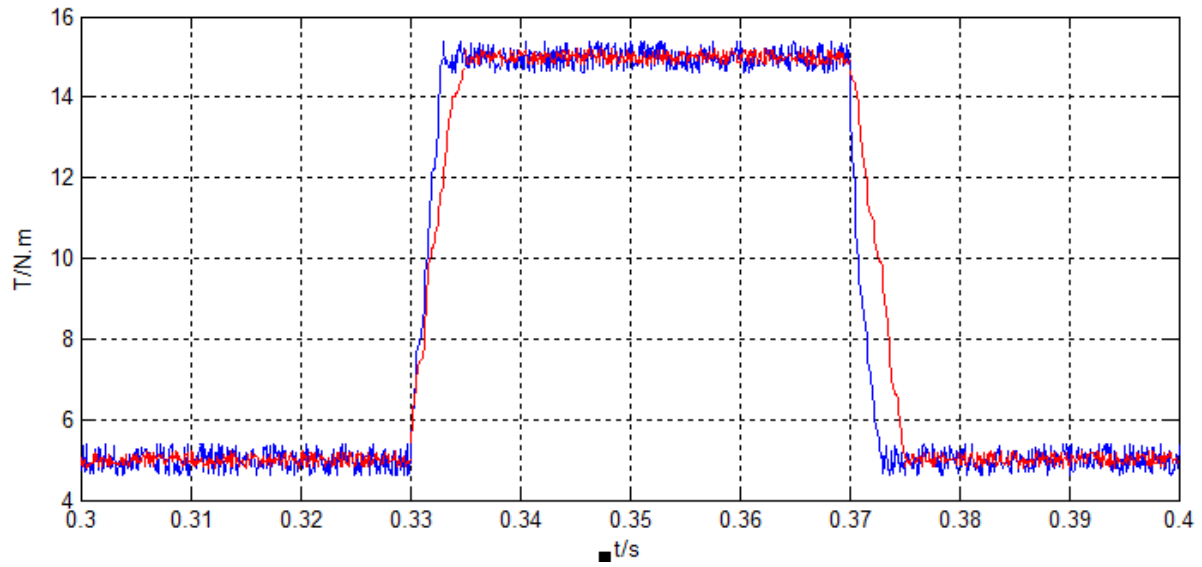

Figure 7. Waveforms of torque dynamic response 
In order to analysis the capability to load sudden changes of different DTC, torque ripple coefficient $(T R F)$ is delimited as function (10) [8].

$$
T R F=\frac{T_{e \max }-T_{e \min }}{T_{\text {eref }}}
$$

$T_{e \max }$ represents the maximum of torque ripple; $T_{e \text { min }}$ represents the minimum of torque ripple; $T_{\text {eref }}$ represents the given torque.

TRF of traditional DTC is about $16 \%$, while $8 \%$ of SVM-DTC, as presented in Figure 7 , latter is obviously better than the former. On the other hand, response time of load sudden changes of traditional DTC is 3 milliseconds, while $5 \mathrm{~ms}$ of SVM-DTC, both can response fast.

\section{Conclusions}

From the analysis of flux speed and torque in steady-state, results show that SVM-DTC proposed in this essay has smaller change scale flux, more stable speed, and smaller rippled torque, when compare to traditional DTC. By the simulation of load sudden changes, response time almost equal, indicates that SVM-DTC presented in this essay can increase the speed of dynamic response while maintaining the good stationary performance as well as the robustness. SVM-DTC proposed in this essay is briefness, easy to carry out and has a certain practical significance in the field of high accuracy and high efficiency PMSM application controlled by DTC.

\section{References}

1. A.Jidin, N.R.N.Idris, A.H.M.Yatim, et al. An optimized switching strategy for quick dynamic torque control in DTC hysteresis based induction machines. J. IEEE Transactions on Industrial Electronics, E 58(8), 3391-3400 (2011).

2. Yanping $\mathrm{Xu}$, Yanru Zhong. Sectors subdivision and duty ratio control combined DTC for PMSM. J. Proceedings of the CSEE, E 29(3), 102-108(2009).

3. D.Casadei, F.Profumo, G.Serra, et al. FOC and DTC: Two variable schemes for induction motors torque control. J. IEEE Transactions on Power Electronics, E 17(5), 779-787(2002).

4. L.Tang, L.Zhong, M.F.Rahman, et al, Novel direct torque controlled interior permanent magnet synchronous machine drive with low ripple in flux and torque and fixed switching frequency. J. IEEE Transactions on Power Electronics, E 19(2), 346-354(2004).

5. Huaqiang Zhang, Xinsheng Wang, et al. Study on direct torque control algorithm based on space vector modulation. J. Electric machines and control, E 06(16),13-18(2012).

6. N.R.N.Idris, A.H.M.Yatim. Direct torque control of induction machines with constant switching frequency and reduced torque ripple. J. IEEE Transactions on Industrial Electronics, E 51(4), 758-767(2004).

7. Dan Sun, Yikang He. Space vector modulated based constant switching frequency direct torque control for permanent magnet synchronous motor. J. Proceedings of the CSEE, E 25(12), 112116(2005).

8. A.Jidin, N.R.N.Idris, A.H.M.Yatim, et al. Simple Dynamic Overmodulation Strategy for Fast Torque Control in DTC of Induction Machines With Constant-Switching-Frequency Controller. J. IEEE Transactions on Industry Applications, E 47(5), 2283-2291(2011). 